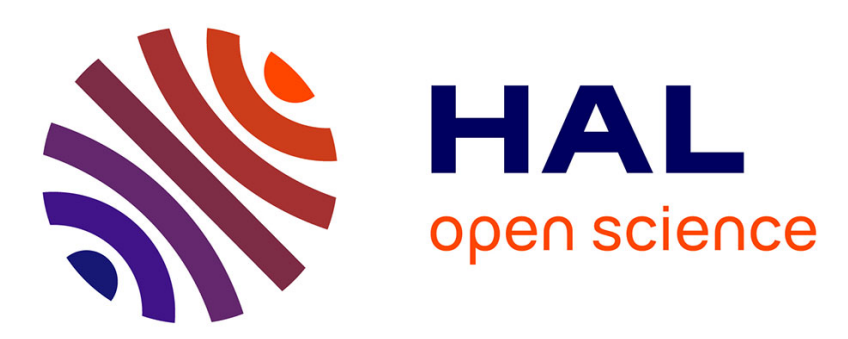

\title{
Force Performance Indexes for Parallel Mechanisms with Actuation Redundancy, Especially for Parallel Wire-Driven Manipulators
}

\author{
Sébastien Krut, Olivier Company, François Pierrot
}

\section{- To cite this version:}

Sébastien Krut, Olivier Company, François Pierrot. Force Performance Indexes for Parallel Mechanisms with Actuation Redundancy, Especially for Parallel Wire-Driven Manipulators. IROS: Intelligent RObots and Systems, Sep 2004, Sendai, Japan. pp.3936-3941. lirmm-00108812

\section{HAL Id: lirmm-00108812 https://hal-lirmm.ccsd.cnrs.fr/lirmm-00108812}

Submitted on 23 Oct 2006

HAL is a multi-disciplinary open access archive for the deposit and dissemination of scientific research documents, whether they are published or not. The documents may come from teaching and research institutions in France or abroad, or from public or private research centers.
L'archive ouverte pluridisciplinaire HAL, est destinée au dépôt et à la diffusion de documents scientifiques de niveau recherche, publiés ou non, émanant des établissements d'enseignement et de recherche français ou étrangers, des laboratoires publics ou privés. 


\section{Force Performance Indexes for Parallel Mechanisms with Actuation Redundancy, especially for Parallel Wire-Driven Manipulators}

\author{
Sébastien Krut ${ }^{\dagger}$ \\ AIST/ISI-CNRS/STIC JRL \\ (Joint Japanese-French Robotics Laboratory) \\ AIST, Central 2, 1-1-1 Umezono \\ Tsukuba, Ibaraki, 305-8568, JAPAN \\ sebastien.krut@aist.go.jp
}

\author{
Olivier Company, François Pierrot \\ LIRMM \\ UMR 5506, CNRS-UM2 \\ 161 , rue Ada \\ 34392 Montpellier Cedex 5, FRANCE \\ $<$ company, pierrot>@lirmm.fr
}

\begin{abstract}
This paper presents new force performance indexes adapted to Parallel Mechanisms with Actuation Redundancy (PMAR) especially parallel wire-driven manipulators (where only tensile forces in cables are possible). Such performance indexes might be used for optimization purpose. At first, the operational force polytope is established. It is the accurate representation of admissible operational forces from the kinetostatic analysis point of view. A way to efficiently compute it is given. The obtained description of the polytope is the same as in [1], but regarding forces. As a consequence the reader is free to use any of the indexes introduced in [1]. From the authors' point of view the "maximum operational isotropic force" is well adapted to characterize parallel wiredriven manipulators' force behavior. The way to compute it is established. At last, this index is computed for several parallel wire-driven manipulators.
\end{abstract}

\section{INTRODUCTION}

Wire-driven manipulators show great performances in terms of dynamics since the weight of the moving parts is reduced to the minimum. As an example the Falcon robot [2][3] (see fig. 1) can reach a $43 g$ acceleration.

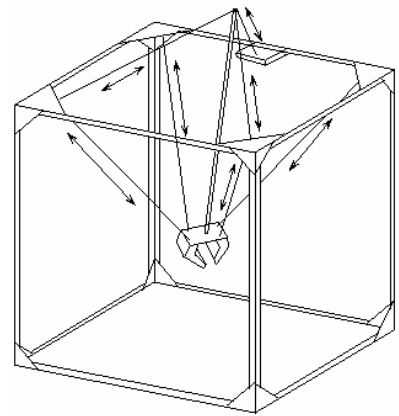

fig. 1 - The Falcon robot

The wire-driven mechanisms we will deal with are different from crane mechanisms. In fact cranes need gravity to maintain positive tension in cables. In our case, we deal with Parallel Mechanisms with Actuation Redundancy (PMAR). Such a redundancy participates to maintain tensile positive in cables.

The Falcon robot is a 7 actuators / 6 degrees-offreedom (dof) mechanism. In order to obtain bigger workspaces, other mechanisms with more actuators were imagined. We can notice the 9 actuators / 6 dof Sacso mechanism [4] developed at Onera, or the force reflecting joystick for telerobotics developed at the NASA [5].

Some mathematical tools were developed to characterize such mechanisms. Kawamura and Ken [6] explained how to verify (with an iterative process) that the tensile in cables is always positive inside the whole workspace. Verhoeven and Hiller [7][8] described with inequalities the domain of the operational space where tensile in cables is higher than a certain level.

Some other approaches based on the rigidity matrix were also developed [9][10], leading to performance indexes. In terms of force [11] and [12] present a way to characterize the admissible domain of operational forces as the union of several ellipsoids.

Another approach has been also proposed by Ngyen in [13], but adapted to the grasping problem. He proposed a graphical method to characterize the force-closure grasps based on the shape of the grasped object. This problem might be transposed to wire-driven manipulators easily (see [14]) while grasping forces must remain always positive such as for tensile forces.

It is claimed here that a good way of characterizing the behavior of wire-driven manipulators is to focus on the operational forces. Two remarks must be done:

- Classical tools, such as the Singular Values Decomposition of the Jacobian matrix or the conditioning number, are not adapted to PMAR [1]. In fact, as soon as there is redundancy, such tools are meaningless regarding performances in the operational space.

- Furthermore, due to redundancy, no corresponding relationship exists between velocity and force transmission (the classical force/velocity duality is not as significant for PMAR as it is for simple Parallel Kinematics Mechanisms), and the study done in [1] regarding velocity transmission has to be extended to force transmission. In fact, if velocities of the actuators must be synchronized, in order for the PMAR to work properly, forces might be chosen freely while they can generate internal forces in the mechanism.

In this paper, at first we will focus on the operational force polytope, which is the exact representation of

\footnotetext{
${ }^{\dagger}$ This work has been started while being at the LIRMM.
} 
attainable forces exerted by the traveling plate (when assuming that actuators forces are bounded). The computation is different from the one introduced in [15], as it is not a simplex algorithm, and it can deal with unsymmetrical force bounds for the actuators. As a consequence it is well adapted for parallel wire-driven manipulators. We will give a systematic representation of the frontiers of the polytope in order to use the specific tools introduced in [1]. As a consequence we will be able to compute all the performances indexes introduced in [1].

In our opinion, a good way to characterize force transmission of parallel wire-driven manipulators is to consider the maximum isotropic operational force, i.e. the force that the traveling plate can always exert in any direction of the operational space. The focus will be put on such an index, and some practical examples will be given.

\section{MODELING OF WIRE-DRIVEN PARALLEL MANIPULATORS AND ANNOTATIONS}

\section{A. Scheme of a typical wire-driven parallel manipulator}

For illustration purpose the planar mechanism shown in fig. 2 will be used. It is a 3 actuator/ 2 dof PMAR, composed of rotational motors pulling on the wires attached to the traveling plate.

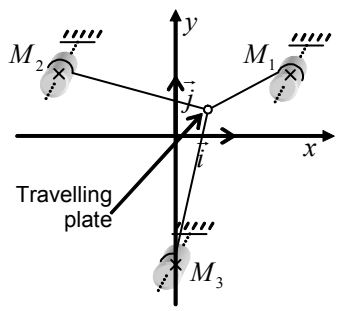

fig. 2 - Geometry of a typical parallel wire-driven mechanism

Note that, for simplification purpose, the effect of gravity is neglected. Gravity introduces a directional bias in the operational force space (refer to [16]). The proposed method might be improved to cope with such a problem.

We will note:

- $\quad m$ the dimension of the joint space (in fig. $2 m=3$ ),

- $n$ the dimension of the operational space $(n=2)$,

- $\boldsymbol{\tau}=\left[\begin{array}{lll}\tau_{1} & \ldots & \tau_{m}\end{array}\right]^{T}$ the joint force vector,

- $\boldsymbol{f}=\left[\begin{array}{lll}f_{1} & \ldots & f_{n}\end{array}\right]^{T}$ the Cartesian force vector.

Formulas will be established for any size of joint and operational spaces, as long as $m>n$.

\section{B. Mapping articular and operational forces through the Jacobian matrix}

The so-called "inverse Jacobian matrix" $\boldsymbol{J}_{\boldsymbol{m}}$ is representative of the linear application linking operational velocities $\dot{\boldsymbol{x}}$ to actuators velocities $\dot{\boldsymbol{q}}$ :

$$
\dot{\boldsymbol{q}}=\boldsymbol{J}_{\boldsymbol{m}} \dot{\boldsymbol{x}} \text {. }
$$

While assuming that no internal power is dissipated in the mechanism, the conservation of power from actuators to the traveling plate $\left(\dot{\boldsymbol{q}}^{T} \boldsymbol{\tau}=\dot{\boldsymbol{x}}^{T} \boldsymbol{f}\right)$ leads to the well known relation:

$$
f=J_{m}{ }^{T} \tau .
$$

The admissible joint force space, i.e. the domain of space in which a point represents a realistic combination of actuator forces, is a rectangular parallelepiped.

Note that we have to generalize this terminology for higher dimension spaces by saying "hyper rectangular parallelepiped".

It is defined by the $2 m$ inequalities:

$$
\left\{\begin{array}{l}
\tau_{i} \geq \tau_{i}^{\text {min }} \\
\tau_{i} \leq \tau_{i}^{\text {max }}
\end{array}, i \in\{1, \ldots, m\},\right.
$$

where:

- $\tau_{i}^{\max }$ is given either by the $i^{\text {th }}$ motor maximum force capability, or by the $i^{\text {th }}$ wire maximum tension capacity. This constitutes a physical limit for the mechanism and is independent of the mechanism load.

- $\tau_{i}^{\text {min }}$ represents the lowest acceptable tensile in cable $\left(\tau_{i}^{\min } \geq 0\right)$. It is often decided that $\tau_{i}^{\min }>0$.

fig. 3 depicts such a domain for $m=3$.

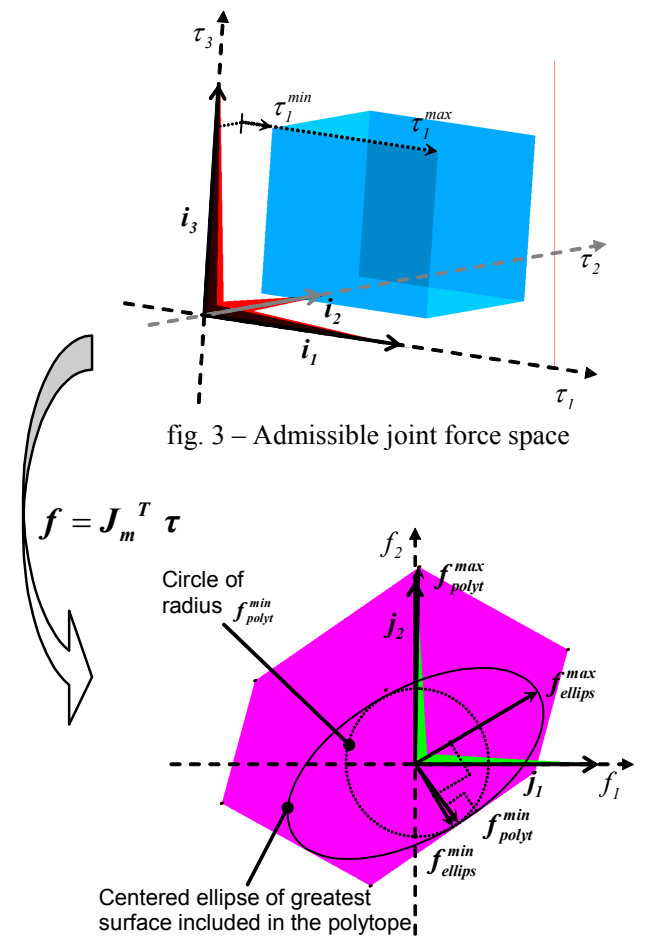

fig. 4 - Admissible operational force space

Mapping the admissible joint space in the operational space (through the Jacobian matrix) leads to the desired polytope. Such a polytope can be observed on fig. 4 for $n=2$. The depicted circle and ellipse are associated to performance indexes. Thus, the radius of the circle corresponds to the largest force that the machine can handle in any direction of the operational space; the deformation of the ellipse is a measure of the force isotropy (but is quite meaningless for parallel wire-driven manipulators, while the operational polytope is not centered on the origin).

Next section will aim at characterizing this operational polytope. A systematic description of its frontiers will be 
given. It will be the same as the one introduced in [1] in order to reuse the exposed performance indexes.

\section{CHARACTERIZATION OF THE OPERATIONAL FORCE POLYTOPE}

The Singular Values Decomposition (SVD) of the Jacobian matrix helps understanding the way the joint rectangular parallelepiped transforms into the operational polytope:

$$
J_{m}^{T}=U S V^{T}
$$

where:

- $\quad \boldsymbol{U}$ and $\boldsymbol{V}$ are orthogonal matrixes of dimension $m \times m$ and $n \times n$ respectively. They represent the rotations from the canonic bases (respectively articular and operational) to the ones adapted to the SVD.

- $\boldsymbol{S}$ is a rectangular matrix of dimensions $n \times m$ containing the singular values $\sigma_{1}, \ldots, \sigma_{n}$. It can be spit in two matrixes:

$$
\boldsymbol{S}=\boldsymbol{S}^{\prime} \boldsymbol{P}
$$

where $\boldsymbol{P}$ is the $n \times m$ projection matrix to the range of $\boldsymbol{J}_{\boldsymbol{m}}{ }^{T}$ and $\boldsymbol{S}^{\prime}$ is the diagonal matrix consisting of the singular values:

$$
\boldsymbol{P}=\left(\begin{array}{lll:l}
1 & & & \\
& \ddots & & 0 \\
& & 1 &
\end{array}\right), \boldsymbol{S}^{\prime}=\operatorname{diag}\left(\sigma_{1}, \ldots, \sigma_{n}\right) .
$$

We will figure out how the joint rectangular parallelepiped transforms when mapped through the different matrixes. To do this let us introduce $\boldsymbol{\tau}_{s}$ the joint vector force expressed in the SVD joint base, $\boldsymbol{\tau}_{\boldsymbol{r}}$ the restriction to the range of $\boldsymbol{J}_{\boldsymbol{m}}{ }^{\boldsymbol{T}}$ of the former vector, and $f_{s}$ the vector of operational forces expressed in the SVD operational base:

$$
\tau \stackrel{V^{T}}{\longrightarrow} \tau_{s} \stackrel{P}{\longrightarrow} \tau_{r} \stackrel{S^{\prime}}{\longrightarrow} f_{s} \stackrel{U}{\longrightarrow} f
$$

fig. 5, fig. 6 and fig. 7 illustrate the depicted transformations.

Our goal will be to point out the equalities which characterize each frontier $\mathcal{S}_{k}$ of the operational polytope. The equality will have to be of the following form in order to be coherent with the formalism introduced in [1]:

$$
\mathcal{S}_{k}=\left\{M \mid \boldsymbol{m}^{T} \boldsymbol{v}_{\boldsymbol{k}}=1\right\},
$$

where $\boldsymbol{m}$ is the vector going from $O$, the origin of the frame, to $M$ a point belonging to the frontier and $\boldsymbol{v}_{\boldsymbol{k}}$ the vector characterizing the side.

Note that such a formalism is always possible except when the side crosses $O$.

We have split the evaluation of the operational polytope in a few steps:

1. finding all vertexes of the joint rectangular parallelepiped,

2. finding their coordinates in the SVD joint base,

3. computing their projection in the range of $\boldsymbol{J}_{\boldsymbol{m}}{ }^{T}$,
4. finding all hyperplanes of the range of $\boldsymbol{J}_{\boldsymbol{m}}{ }^{T}$ linking all possible combinations of vertexes' projection.

5. Seeking among those planes in order to keep only the frontiers of the polytope,

6. mapping those chosen planes into the operational space.

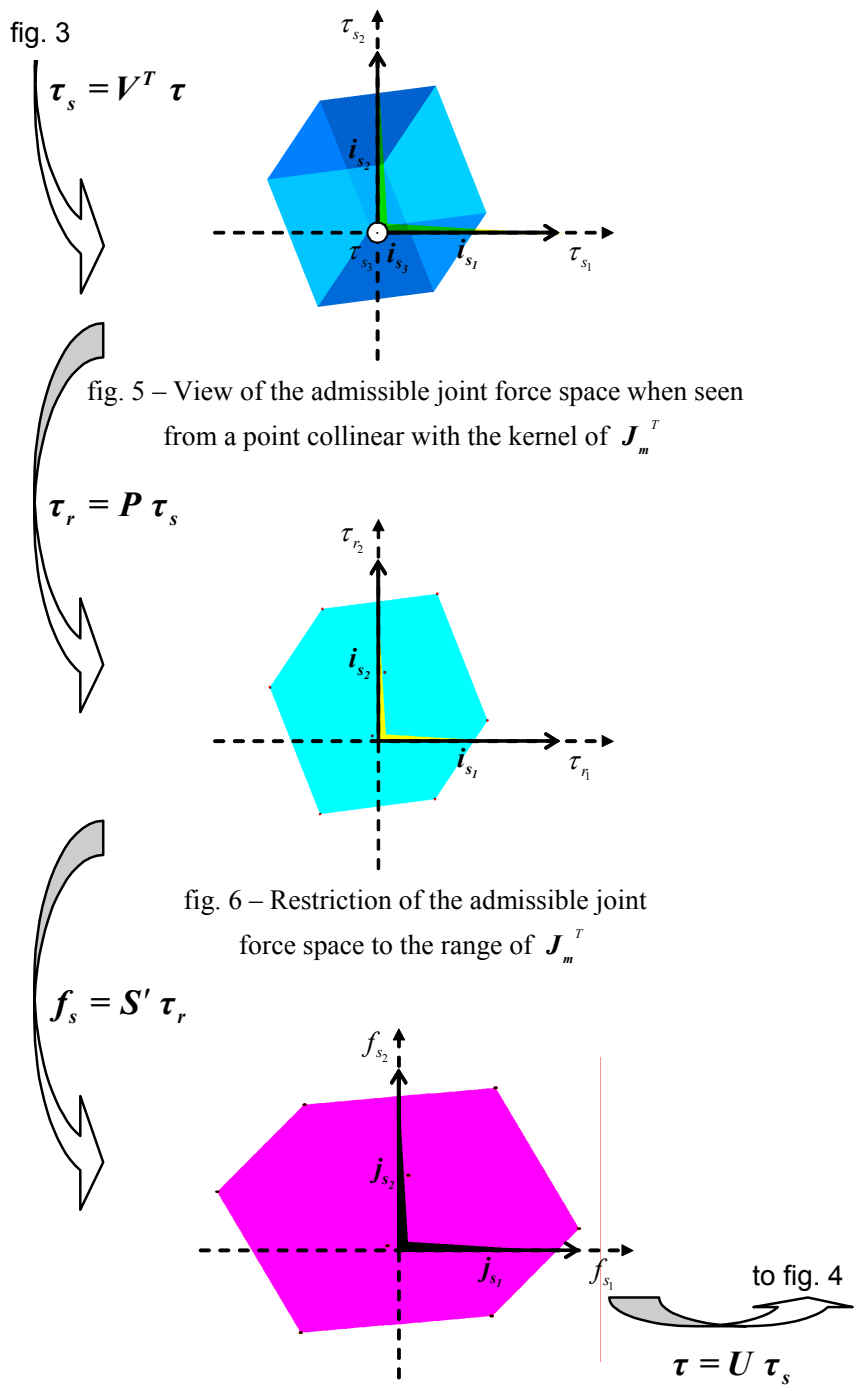

fig. 7 - View of the admissible operational force space directed by the vectors of the SVD operational base

Step 1

The admissible joint space has $2^{m}$ vertexes: $\boldsymbol{\tau}_{\text {vertex }}$, $l \in\left\{1, \ldots, 2^{m}\right\}$. One way to compute them efficiently is to write the binary representation of $l-1$ and to assume that when the $i^{\text {th }}$ digit of it is equal to 1 , the $i^{\text {th }}$ coordinate of $\boldsymbol{\tau}_{\text {vertex }_{l}}$ should be $\tau_{i}^{\max }$, otherwise $\tau_{i}^{\min }$.

Example:

$m=3 \rightarrow$ there is $2^{3}=8$ corners (see fig. 3), and corner number 5 can be define as:

$$
\begin{aligned}
& 5-1=4=0000100 \underbrace{}_{\text {binary }} \\
& \boldsymbol{\tau}_{\text {vertex }_{5}}=\left[\begin{array}{llllllll}
\tau_{1}^{\text {min }} & \tau_{2}^{\min } & \tau_{3}^{\text {min }} & \tau_{4}^{\min } & \tau_{5}^{\min } & \tau_{6}^{\max } & \tau_{7}^{\min } & \tau_{8}^{\min }
\end{array}\right]^{T}
\end{aligned}
$$

This vector is depicted in the canonic joint base. 
$\underline{\text { Step } 2}$

$$
\tau_{s_{\text {vertex }}}=V^{T} \tau_{\text {vertex }}
$$

$\tau_{s_{\text {vertex }}}$ is the representation of $\tau_{\text {vertex }_{l}}$ in the SVD joint base.

$\underline{\text { Step } 3}$

$$
\tau_{r_{\text {vertex }}}=\boldsymbol{P} \tau_{s_{\text {vertex }}}
$$

$\boldsymbol{\tau}_{r_{\text {verex }}}$ is a $n$ size vector. It represents the projection of $\boldsymbol{\tau}_{s_{\text {vertex }}}$ in the range of $\boldsymbol{J}_{\boldsymbol{m}}{ }^{\boldsymbol{T}}$. There is still $2^{m}$ vertexes like this one $\left(l \in\left\{1, \ldots, 2^{m}\right\}\right)$.

Step 4

While each frontier of the polytope is an hyperplane, and while an hyperplane is of dimension $n-1$, each frontier can be characterized with only one linear equation (linking the coordinates of the hyperplane). The obtained equation will be of the following form:

$$
p^{T} u_{k}=1,
$$

where $\boldsymbol{u}_{\boldsymbol{k}}$ is the characteristic vector of hyperplane $k$, and $\boldsymbol{p}$ the column vector associated to point $P$ belonging to the hyperplane.

Note that in the case of fig. 3 each hyperplane is a line.

While $\boldsymbol{u}_{\boldsymbol{k}}$ is of dimension $n, n$ independent linear equations are required to establish it. The proposed method consist in choosing $n$ distinct vertexes' projection $\boldsymbol{\tau}_{\boldsymbol{r}_{\text {vertex }}}$ among the $2^{m}$ ones, and expressing the fact that they belong to the hyperplane, that is to say:

$$
\boldsymbol{\tau}_{\boldsymbol{r}_{\text {verex } x}}{ }^{T} \boldsymbol{u}_{\boldsymbol{k}}=1 \text {. }
$$

We can count $C_{2^{m}}^{n}$ different ways of choosing those points.

Note: $C_{2^{m}}^{n}$ represents the number of combinations of $n$ elements among $2^{m}$.

Thus, $C_{2^{m}}^{n}$ linear systems might be obtained. Each system can be written as follows:

$$
\left[\begin{array}{cc}
\boldsymbol{\tau}_{\boldsymbol{r}_{\text {vertex }_{1}}} \\
\\
\vdots \\
\boldsymbol{\tau}_{\boldsymbol{r}_{\text {verex }_{H_{n}}}}{ }_{T}
\end{array}\right] \boldsymbol{u}_{k}=\left[\begin{array}{c}
1 \\
\vdots \\
1
\end{array}\right],
$$

with $l_{i} \in\left\{1, \ldots, 2^{m}\right\}$, depending which of the $C_{2^{m}}^{n}$ combinations we are dealing with $(i \in\{1, \ldots, n\})$ and $\left.k \in\left\{1, \ldots, C_{2^{m}}^{n}\right\}\right)$.

Let us note $\boldsymbol{T}_{k}$ the characteristic matrix of this equation system:

$$
\boldsymbol{T}_{\boldsymbol{k}}=\left[\begin{array}{cc}
\boldsymbol{\tau}_{\boldsymbol{r}_{\text {errex }_{1}}} & T \\
& \vdots \\
\boldsymbol{\tau}_{\boldsymbol{r}_{\text {verex }}} & T
\end{array}\right] .
$$

The resolution of the $n$ equations/ $n$ unknowns system (12) leads to vector $\boldsymbol{u}_{k}$ characteristic of hyperplane $k$ :

$$
\boldsymbol{u}_{k}=\boldsymbol{T}_{k}^{-1}\left[\begin{array}{lll}
1 & \ldots & 1
\end{array}\right]^{T} .
$$

We must notice that for some combinations of points, system (12) is not a Cramer system $\left(\operatorname{det}\left(\boldsymbol{T}_{k}\right)=0\right)$. Thus, vector $\boldsymbol{u}_{k}$ can not be determined. This phenomenon occurs when two corners' projection are identical $\left(\boldsymbol{\tau}_{\boldsymbol{r}_{\text {verex }_{i}}}=\boldsymbol{\tau}_{\boldsymbol{r}_{\text {vertex }_{j}}}, i, j \in\left\{1, \ldots, 2^{m}\right\}\right)$ or when they are aligned with the origin $O\left(\exists k \in \mathbb{R}^{*} \mid \boldsymbol{\tau}_{\boldsymbol{r}_{\text {enrex }_{i}}}=k \boldsymbol{\tau}_{\boldsymbol{r}_{\text {errex }_{j}}}\right)$. In the first situation, there is no need to matter of vector $\boldsymbol{u}_{k}$, while this combination of points leads to no hyperplane. In the second situation, a hyperplane might exist. This hyperplane crosses the origin $O$. Whether this hyperplane is not a frontier, or it witnesses a singular posture. The final algorithm takes into account such a difficulty.

This step leads to $q$ vectors $\boldsymbol{u}_{k}, \quad q \leq C_{2^{m}}^{n}$, $k \in\{1, \ldots, q\}$. Each vector characterizes a correct hyperplane, that is to say a possible frontier for the polytope (see fig. 8).

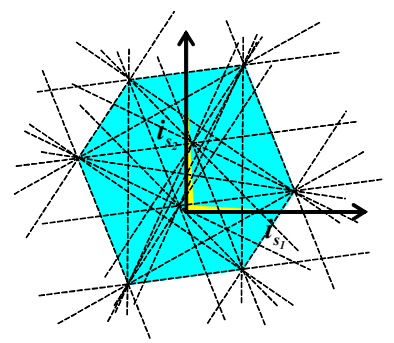

fig. 8 - Representation of all the possible frontiers for the polytope Step 5

How to find the frontiers of the polytope among the $q$ hyperplanes? By noticing that when a hyperplane is a frontier all the vertexes are located only on one side of the hyperplane.

Mathematically this can be written:

$$
(\text { BorderType1 }=\text { TRUE) OR }(\text { BorderType } 2=\text { TRUE })
$$
with:

$$
\begin{aligned}
& \text { BorderTypel }=\left(\left(\boldsymbol{\tau}_{\boldsymbol{r}_{\text {vertex }_{1}}} \boldsymbol{T}_{\boldsymbol{k}} \leq 1\right) \ldots \text { AND }\left(\boldsymbol{\tau}_{\boldsymbol{r}_{\text {vertex }_{2} m}} \boldsymbol{u}_{\boldsymbol{k}} \boldsymbol{u}_{\boldsymbol{k}} \leq 1\right)\right) \text {, } \\
& \text { BorderType2 }=\left(\left(\boldsymbol{\tau}_{\boldsymbol{r}_{\text {verex }_{1}}} \boldsymbol{T}_{\boldsymbol{k}} \geq 1\right) \ldots \text { AND }\left(\boldsymbol{\tau}_{\boldsymbol{r}_{\text {vertex }_{2}{ }^{m}}} \boldsymbol{T}_{\boldsymbol{k}} \geq 1\right)\right) \text {. }
\end{aligned}
$$

When condition (15) is fulfilled, it can be certified that $\boldsymbol{u}_{\boldsymbol{k}}$ characterizes a frontier of the polytope. Among all the $q$ correct hyperplanes, only $r$ constitute sides for the polytope $(r \leq q)$.

Once the borders are established, it is still to be verified that the origin of the frame belongs to the polytope. In fact, if $O$ is located outside, it means that in this particular position the mechanism can't even balance a null perturbation, i.e. that this specific arrangement is unachievable. This test is done by checking that all vertexes projections are located below the considered border (border is of type 1), and by replaying this test for every border.

At the end, a complete mathematical description of the sought polytope is established: 


$$
\left\{\begin{array}{cc}
p^{T} & u_{1} \leq 1 \\
& \cdots \\
p^{T} & u_{r} \leq 1
\end{array}\right.
$$

This polytope -restriction of the admissible joint force space to the range of $\boldsymbol{J}_{\boldsymbol{m}}{ }^{T}$ - is fully characterized by a set of $r$ inequalities, where $\boldsymbol{p}$ is the vector associated to point $P$, a point belonging to the polytope, and $\boldsymbol{u}_{i}, i \in\{1, \ldots, r\}$ the vectors characterizing each frontier of the polytope.

\section{$\underline{\text { Step } 6}$}

Mapping the polytope in the operational space is done very easily by mapping each frontier. With next formula (taken from [1]), we recall the way to compute the frontiers' characteristic vectors:

$$
v_{k}=S^{\prime} u_{k}
$$

$v_{k}$ represents the characteristic vector of side $k$ in the operational SVD base.

Finally, the force operational polytope is obtained. It is depicted by:

$$
\left\{\begin{array}{c}
\boldsymbol{m}^{T} v_{1} \leq 1 \\
\cdots \\
\boldsymbol{m}^{T} v_{r} \leq 1
\end{array}\right.
$$

Such a representation is the one introduced in [1]. All the proposed indexes might be used to characterize such a polytope.

\section{COMPUTATION OF THE LARGEST ISOTROPIC FORCE FOR A PARALLEL WIRE-DRIVEN MANIPULATOR}

As proposed in the paper introduction the maximal isotropic force might be a good representation of the manipulator's behavior. It represents the intensity of the largest perturbation that the nacelle can balance in any direction in the operational space. Graphically it corresponds to the radius of the largest circle included in the operational force polytope (see fig. 4).

What will be done is to recall (taken from [1]) very briefly the way to obtain such characteristics. Afterward, we will apply the depicted criteria to a particular parallel wire-driven robot.

\section{A. Recall of the method}

The way to compute the lowest operational force $\boldsymbol{f}_{\text {polyt }}^{\text {min }}$ might be sum up with this single formula:

$$
\boldsymbol{f}_{\text {polyt }}^{\min }=\min _{i \in\{1, \ldots, r\}} \frac{1}{\left\|\boldsymbol{v}_{i}\right\|}
$$

(Refer to [1] for any explanations.)

It represents the largest force that the machine can handle in any direction of the operational space.

\section{B. Case study}

We implemented the above algorithm with Matlab for validation purpose. We decided to characterize the behavior of the planar parallel wire-driven manipulator depicted in fig. 2, by computing the lowest operational force in its whole workspace.
The center of the three actuators can be depicted by their polar coordinates:

$$
\begin{gathered}
\theta_{1}=30^{\circ}, \theta_{2}=150^{\circ}, \theta_{3}=270^{\circ}, \\
l_{i}=l=1000 \mathrm{~mm},
\end{gathered}
$$

where $\theta_{i}$ is the angle between the $x$ axis and the motor limb, and $l_{i}$ is the distance taken from the origin, $i \in\{1,2,3\}$.

The Jacobian matrix $\boldsymbol{J}_{\boldsymbol{m}}$ is:

$$
\boldsymbol{J}_{\boldsymbol{m}}=\left(\begin{array}{cc}
a_{1_{x}} /\left\|\boldsymbol{a}_{1}\right\| & a_{1_{y}} /\left\|\boldsymbol{a}_{1}\right\| \\
a_{2_{x}} /\left\|\boldsymbol{a}_{2}\right\| & a_{2_{y}} /\left\|\boldsymbol{a}_{2}\right\| \\
a_{3_{x}} /\left\|\boldsymbol{a}_{3}\right\| & a_{3_{y}} /\left\|\boldsymbol{a}_{3}\right\|
\end{array}\right),
$$

where $\boldsymbol{a}_{i}$ represents the vector joining $M$ (coordinates $x$ and $y$ ) the position of the traveling plate, to $M_{i}$ the position of motor $i$, in the canonic operational base $\left(a_{i_{x}}\right.$ and $a_{i_{y}}$ are respectively its coordinates along the abscise and the ordinate):

$$
\boldsymbol{a}_{i}=\left(\begin{array}{c}
l \cos \theta_{i}-x \\
l \sin \theta_{i}-y
\end{array}\right)
$$

Note that in this particular case, a closed-form Jacobian matrix has been derived. But since the index computation does not require a closed-form this computation can be done numerically.

While scanning the operational space, the Jacobian matrix is computed and used to establish the lowest operational force for every particular position.

Actuator forces were required to be in the range $[50,500]$ : that is to say $\tau_{i} \geq 50$ and $\tau_{i} \leq 500$ for $i \in\{1,2,3\}$.

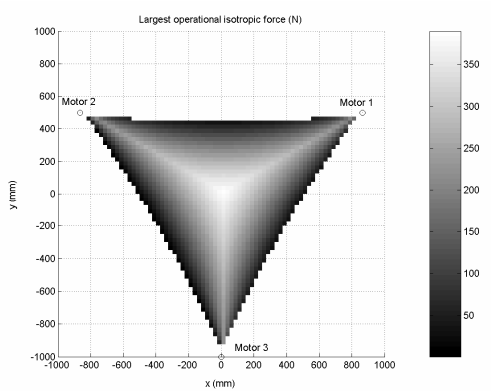

fig. 9 - Representation of the largest isotropic force for a 3 actuators $/ 2$ dof parallel wire-driven manipulator

fig. 9 represents the intensity of the isotropic force for all positions of the ending part in the workspace theoretical workspace is computed with the same algorithm while it corresponds to the domain of space where $\boldsymbol{f}_{\text {polyt }}^{\text {min }} \geq 0$. For clearness purpose the domain $\boldsymbol{f}_{\text {polyt }}^{\text {min }}<0$ has been drawn in white.

\footnotetext{
\$ See video for better understanding. The left part of the animation represents the admissible joint force space (a cube) when seen from a point collinear with the kernel of $\boldsymbol{J}_{\boldsymbol{m}}{ }^{T}$. The right side represents the operational force polytope centered on the nacelle of the mechanism. The radius of the drawn circle corresponds to the largest isotropic force $\boldsymbol{f}_{\text {polyt }}^{\min }$.
} 
With such results, we are able to manage an optimization process in order to find the best geometrical parameters (for example we might want the lowest operational force to be larger than a given limit, in the required workspace).

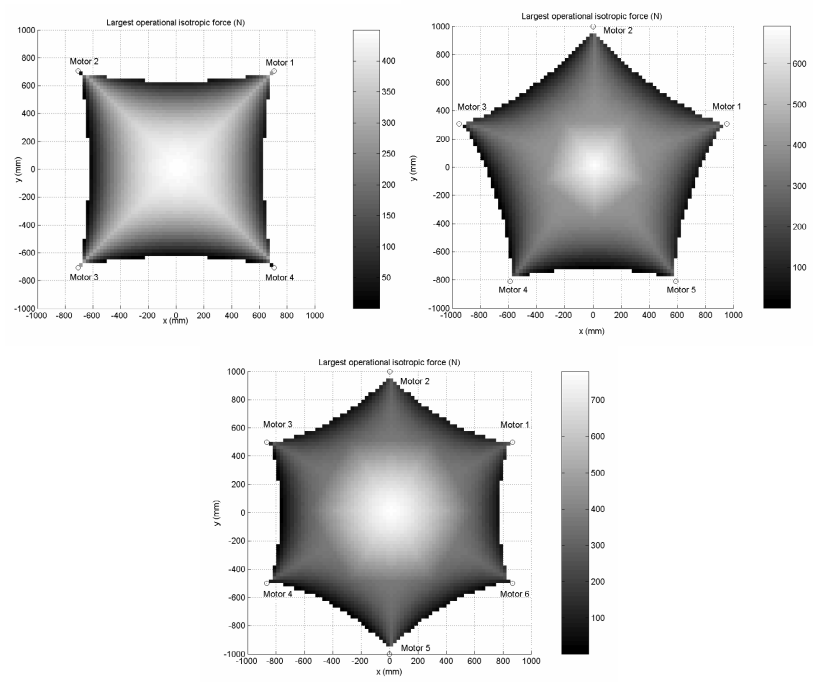

fig. 10 - Representation of the largest isotropic force for $n$ actuators/2 dof parallel wire-driven manipulators, $n \in\{4,5,6\}$

fig. 10 represents the intensity of the largest isotropic force for three different parallel wire-driven manipulators having 2 dof. The $n$ actuators, $n \in\{4,5,6\}$, are regularly dispatched on a circle of radius $l$.

It can be observed that the bigger the number of actuators is, the larger the intensity of the maximum isotropic force becomes, in the middle of the workspace. Furthermore, the workspace for such mechanisms is not exactly a triangle, a square, a pentagon, a hexagon, while strength in cables must remain higher than a certain level bigger than zero.

\section{Extensions}

The authors would like to insist on three particular aspects:

- If this method is well adapted to wire-driven parallel manipulators, it can also cope with normal PMAR, where tensile might be negative (for example $\tau_{i}^{\min }=-\tau_{i}^{\max }$ ).

- We did focus on the maximum isotropic force, but as in [1], we can also compute the centered ellipse with the largest surface included inside the operational force polytope (see fig. 4); it might be another way to characterize the behavior of such mechanisms.

- In this paper the algorithm is presented linearly, but there are ways to optimize it, for example by mixing a few steps in one single stage. Anyway, optimization is not required while this computation has no real-time purpose.

\section{CONCLUSION}

In this paper, we presented a mathematical technique to establish the force characteristics of PMAR, especially wire-driven parallel manipulators. This technique, based on the Jacobian matrix and on the use of the force polytope, leads to performance indexes. It has been used to compute the largest isotropic force, that is to say the maximal force a parallel wire-driven robot can handle, in any direction of its operational space. Such a characteristic might be used for optimization purpose.

\section{ACKNOWLAGEMENT}

This work has been partially supported by the Japan Society for the Promotion of Science (JSPS).

\section{REFERENCES}

[1] Krut S., Company O. and Pierrot F., "Velocity Performance Indexes for Parallel Mechanisms with Actuation Redundancy", Int. J. of Robotica, Cambridge University Press, Vol. 22, Part 2, March-April 2004.

[2] Kawamura S., Choe W., Tanaka S. and Pandian S.R., "Development of an Ultrahigh Speed Robot FALCON using Wire Drive System", in Proc. of IEEE ICRA: Int. Conf. on Robotics and Automation, Nagoya, Japan, pp. 215-220, 1995.

[3] Kawamura S., Kino H. and Choe W., "High-speed Manipulation by using Parallel Wire-driven Robots", in Int. J. of Robotica, Cambridge University Press, Vol. 18, Part 1, pp.13-21, January/February 2000.

[4] Lafourcade, P., Libre M. and Reboulet, C., "Design of a Parallel Wire-driven Manipulator for Wind Tunnels", in Proc. of Workshop on Fundamental Issues and Future Research Directions for Parallel Mech. and Manipulators, Quebec City, Quebec, Canada, October 2002.

[5] Lindemann R. and Tesar D., "Construction and Demonstration of a 9-String 6-DOF Force Reflecting Joystick for Telerobotics", in Proc. of NASA International Conference on Space Telerobotics, No. 4, pp. 55-63, 1989.

[6] Kawamura S. and Ken H., "A New Type of Master Robot for Teleoperation Using a Radial Wire Drive System", in Proc. of IEEE/RSJ International Conference on Intelligent Robots and Systems, Yokohama, Japan, July 26-30, 1993.

[7] Verhoeven R. and Hiller M., "Estimating the Controllable Workspace of Tendon-based Stewart Platforms", in Proc. of ARK International Symposium on Advances in Robot Kinematics, pp. 277-284, Portoroz, Slovenia, 2000.

[8] Verhoeven R. and Hiller M., "Tension Distribution in Tendon-based Stewart Platforms", in Proc. of ARK International Symposium on Advances in Robot Kinematics, Caldes de Malavella, Spain, 2002.

[9] Choe W., Kino H., Katsuta K. and Kawamura S., "A Design of Parallel Wire Driven Robots for Ultrahigh Speed Motion Based on Stiffness Analysis", in Proc. of the Japan-USA Symposium on Flexible Automation, Vol.1, pp.159-166, Boston, USA, July 1996.

[10] Verhoeven R., Hiller M. and Tadokoro S., "Workspace of tendondriven Stewart platforms: Basics, classification, details on the planar 2dof class", in Proc. of the 4th. International Conference on Motion and Vibration Control MOVIC'98, Vol. 3, pp. 871-876, 1998.

[11] Takeda Y. and Funabashi H., "Kinematic Synthesis of Spatial Inparallel Wire-driven Mechanism with 6 Degrees of Freedom with 8 Force Transmissibility", in Proc. of DETC-ASME Design Engineering Technical Conf., Baltimore, Maryland, USA, September 10-13, 2000.

[12] Shen Y., Osumi H. and Arai T., "Manipulability Measures for Multi-wire Driven Parallel Mechanisms", in Proc. of IEEE Int. Conf. on Industrial Technology, pp. 550-554.

[13] Nguyen V.-D., "Constructing Force-Closure Grasps", in IJRR: Int. Journal of Robotics Research, Vol. 7, No. 3, pp.3-16, June 1988.

[14] Ebert-Uphoff I. and Voglewede P. A., "On the Connections Between Cable-Driven Robots, Parallel Manipulators and Grasping", in Proc. of IEEE ICRA'04: International Conference on Robotics and Automation, New Orleans, LA, USA, April 26-Mai 1, 2004

[15] Chiacchio P., Bouffard-Vercelli Y. and Pierrot F., "Evaluation of Force Capabilities for Redundant Manipulators", in Proc. of IEEE ICRA'96: International Conference on Robotics and Automation, Minneapolis, Minnesota, USA, April 1996.

[16] P. Chiacchio, S. Chiaverini, L. Sciavicco, and B. Siciliano, "Influence of gravity on the manipulability ellipsoid for robot arms", in Journal of Dynamic Systems, Measurement and Control, vol. 114, no. 4, pp. 723-727, 1992. 